\title{
Response Analysis of a Scraper Conveyor under Chain Faults Based on MBD-DEM-FEM
}

\author{
Zisheng Wang ${ }^{1,2}$ - Bo Li ${ }^{1,2}$ - Chao Liang1,2 - Xuewen Wang $1,2,{ }^{*}-$ Jiahao Li $^{1,2}$ \\ ${ }^{1}$ Taiyuan University of Technology, College of Mechanical and Vehicle Engineering, China \\ 2 Shanxi Key Laboratory of Fully Mechanized Coal Mining Equipment, China
}

To tackle the difficulty in obtaining the response data of chain and bulk coal under chain faults, this paper uses a new method for the fault simulation of scraper chains based on the coupling of multi-body dynamics (MBD), discrete element method (DEM), and finite element method (FEM). With the force and stacking angle as response values, the contact parameters of bulk coal were revised using a rotary transport test. The simplified DEM-MBD model was verified from the resistance using the point-by-point method. The static structure model of the chain was verified by the chain tensile experiment. The DEM-MBD coupling results show that when the chain is stuck or broken, the dynamic properties of the chain and bulk coal fluctuate sharply, and the wear of the medium plate increases. Based on the DEM-MBD coupling results and the DEM-FEM unidirectional coupling, the stress, strain and life were acquired, and were verified experimentally. Regarding the fracture, the Plackett-Burman test was used to determine that the crack depth, initial angle, and tensile load significantly affect the stress intensity factor (SIF). The quadratic model between significant factors and SIF was constructed using the response surface method, which provides a reference for the simulation of the scraper conveyor, the fault mechanism, and the optimization of the design of the chain.

Keywords: multi-body dynamics, discrete element method, finite element method, scraper chain fault, stress intensity factor, response surface method

\section{Highlights}

- A method for the fault simulation of a scraper conveyor based on the MBD-DEM-FEM was proposed.

- The contact parameters of bulk coal were revised from the force and stacking angle using the response surface method.

- The response data of the chain and bulk coal under chain faults were obtained.

- The interactions between factors on stress intensity factor were analysed using the response surface method.

\section{INTRODUCTION}

As the core structure of the scraper conveyor, the scraper chain is subject to frequent faults, which seriously threatens the safety of workers and the mining efficiency of coal mines [1]. For satisfying the high requirements of intelligent coal mines for scraper chains, it is not feasible to perform fault tests on actual scraper conveyors or scaled models to obtain real-time data. This method is costly, and it is difficult to extract data. Consequently, safety cannot be guaranteed due to the uncertainty of fault. The simulation method based on computer aided design has low cost and high safety factor, and can reproduce the fault state realistically. The common simulation methods include finite element method (FEM), multi-body dynamics (MBD), and discrete element method (DEM).

FEM is based on the idea of discretization and numerical approximation to solve problems [2]. Based on the simplified chain transmission model, researchers have obtained the stress, strain, [3] and [4] and fatigue life [5] of chains under different conditions through transient dynamics or static structural analysis. However, the finite element analysis (FEA) based on the simplified model puts higher demands on the boundary conditions. The accuracy of boundary conditions set only by finite element software is not high but can be enhanced by coupling with DEM [6] and MBD.

MBD focuses on the kinematic and dynamic behaviour of multi-body systems and the mechanical interaction between parts. The velocity and vibration characteristics of the scraper chain under chain stuck and breaking faults were gained by Jiang et al. [7]. Xie et al. [8] discussed the torsional pendulum and vibration characteristics of the chain under chain stuck fault. With the maturity of flexible technology, scholars have made the chain flexible to obtain the stress and deformation [9] and [10]. Previous studies rarely considered the role of bulk coal or only replaced it with a constant load, ignoring the distribution and time-varying characteristics of bulk coal. However, the force and movement of bulk coal under fault conditions may worsen the fault and accelerate the failure of parts.

Considering the discrete characteristics of bulk coal [11], DEM has been applied to research the transport state of scraper conveyors [6] and [12] and the failure of key parts [13] and [14]. Researchers have improved the reliability of simulation by modifying 
parameters [15] and [16] and coupling simulation. The MBD-DEM bidirectional coupling model of scraper conveyor was established by Zhang et al. [17] to realize real-time sharing of information between bulk coal and rigid bodies.

The surface cracks caused by the synergy of complex factors such as wear, corrosion, and deformation are reasons for the fatigue fracture of the chain [18]. It is necessary to discuss the initiation and propagation of cracks. The stress intensity factor (SIF) is a fracture parameter that characterizes the stress field at the crack front, which is affected by the size and shape of the crack. Xue et al. [19] investigated the effects of crack depth and position on fracture parameters through the single factor test, which does not involve the interaction between the factors controlling the fracture of chains. The response surface method (RSM) is an optimization method combining mathematical statistics and modelling [20]. The effects of interaction on the response value can be investigated.

It can be seen that the advantages of each method can compensate for the deficiencies of other methods, so this study used a new method for the fault simulation of a scraper conveyor based on the coupling of MBD-DEM-FEM. First, the MBD-DEM coupling model was established and verified to gain the response characteristics of chain and bulk coal under chain stuck and breaking faults. Combining the above results and DEM-FEM coupling, this study obtained the stress, strain, and life of the chains. For the fracture failure, through single factor testing and response surface testing, the single and interactive effects of crack depth, initial angle, and tensile load on the SIF were investigated. This provides a reference for the simulation of scraper conveyors, and the fault mechanism and optimization design of the chain.

\section{SIMULATION MODELS AND TEST}

\subsection{Dynamic Model}

The coupling simulation with the complete length of the scraper conveyor (SGZ880/800) is not workable [17], so the dynamic model is simplified as necessary. When the chain is stuck or broken, the chain far from the fault point will not respond in a short time. Therefore, only three middle troughs, scraper chains, and drive sprockets are retained; the retarder, hydraulic coupler, and drive motor are removed, and the bottom chute for the return of the chains is added. The simplified model is 5 metres long, as shown in Fig. 1. In the pre simulation, the sprockets drove the scraper chains to run smoothly, which satisfied the desired motion characteristics. The kinematic pairs and contacts of components were set up in RecurDyn dynamic software. The stiffness and damping coefficients of contacts between parts were adjusted by pre-simulation to be $600000 \mathrm{~N} \cdot \mathrm{mm}^{-1}$ and 1000 $\mathrm{N} \cdot(\mathrm{mm} / \mathrm{s})^{-1}$.

\subsection{Construction and Modification of Bulk Coal}

\subsubsection{Model of Coal Particle}

The three typical shapes of coal particles were built by the spherical filling method [21], shown in Fig. 2 . In order to obtain the contact characteristics of the bulk coal with the scraper chain and the medium plate more accurately, coal with large $(50 \mathrm{~mm}$ to $100 \mathrm{~mm})$, medium ( $25 \mathrm{~mm}$ to $50 \mathrm{~mm}$ ), and small $(13 \mathrm{~mm}$ to 25 $\mathrm{mm}$ ) sizes was generated by the particle factory fixed above the machine tail, and the mass proportions of three sizes are $0.25,0.4$, and 0.35 .

Hertz-Mindlin (no slip) [22] and Hertz-Mindlin with Archard wear [14] were chosen for the contact models between particles and between particles and rigid bodies, respectively. The intrinsic parameters

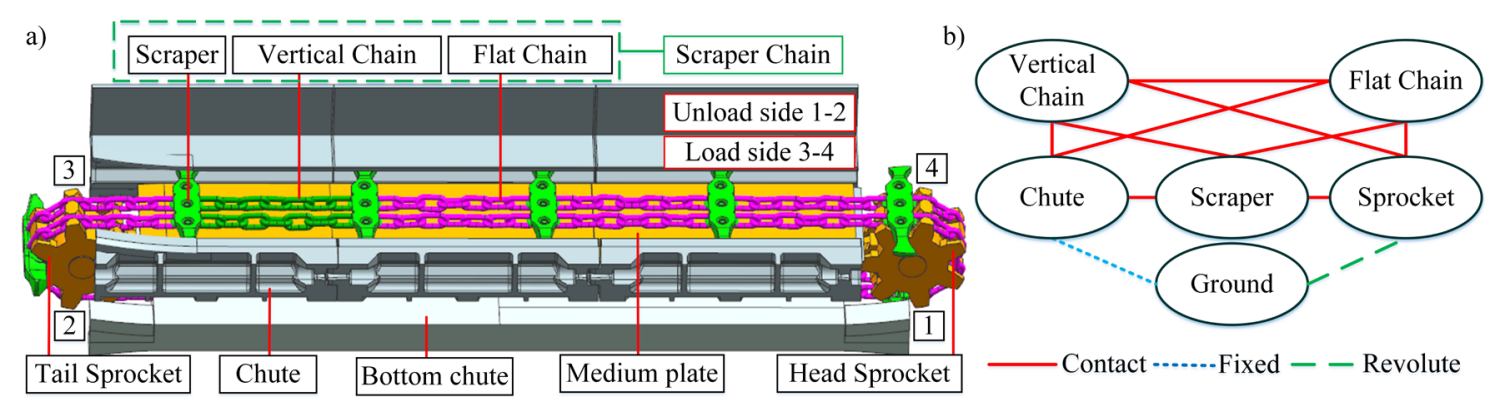

Fig. 1. The mechanical relationship of simplified model; a) simplified model; and b) mechanical relationship 
measured by the group members through the designed experiments are listed in Table 1 [16].

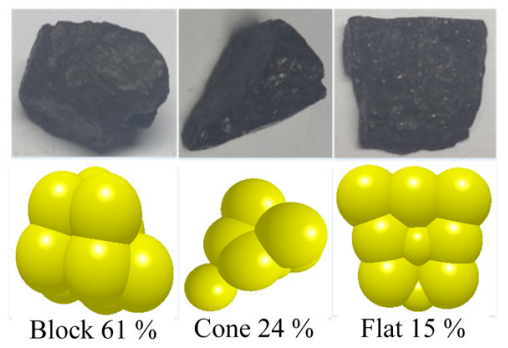

Fig. 2. The shapes and mass proportions of particles

Table 1. The intrinsic parameters

\begin{tabular}{lcc}
\hline Parameters & Steel & Coal \\
\hline Shear modulus [MPa] & 800 & 470 \\
\hline Poisson's ratio & 0.3 & 0.3 \\
\hline Density $[\mathrm{kg} \cdot \mathrm{m}-3]$ & 7850 & 1229 \\
\hline
\end{tabular}

\subsubsection{Modification of Contact Parameters}

In current research, the calibration tests of coal particle parameters take the stacking angle at rest as the response variable. During the conveying process, the behaviour of bulk coal is not only macroscopic accumulation on the chutes, but also the mechanical interaction with the scraper conveyor. Therefore, it is necessary to modify the contact parameters of coal particles through the bulk coal transportation test with force and accumulation as the response variables.

Using the actual scraper conveyor to carry out the transportation test is not feasible. Based on the transport principle, the rotary transport testing machine shown in Fig. 3a was designed. The upper sample has a wedge structure with the same slope as the scraper, as shown in Fig. 3e, and the material of the upper sample is $42 \mathrm{CrMo}(\mathrm{C}: 0.38 \%$ to $0.45 \%$ ), which is the same as the scraper. The lower samples are six fan-shaped plates with positioning holes, as shown in Fig. 3f, and the material of the lower samples is $16 \mathrm{Mn}(\mathrm{C}: 0.13 \%$ to $0.19 \%)$, which is commonly used in the medium plate. The three-dimensional force sensor and the upper sample are fixed with the rack through the clamp, and the lower samples are fixed with the trough through the positioning holes. After the bulk coals with the required mass and size are paved in the trough, the motor drives the trough to rotate at a uniform angular velocity of $10 \mathrm{rad} \cdot \mathrm{s}^{-1}$. The linear velocity of the position where the upper sample is located is the same as that of the scraper chain $\left(1.1 \mathrm{~m} \cdot \mathrm{s}^{-1}\right)$, as shown in Fig. 3c. The bulk coals are pushed by the upper sample and accumulate around the sample, which is consistent with the principle of scraper transportation in actual work, as shown in Fig. $3 \mathrm{c}$. The force on the upper sample when pushing the bulk coals was measured through the force sensor, as shown in Fig. 4. The stacking angle obtained using an image-processing program written in Matlab is $38.59^{\circ}$.

Through Plackett-Burman and response surface tests, the quadratic polynomials between force, stacking angle and significant terms were established. The contact parameters obtained by solving the polynomials with the experimental force and stacking angle as the target values are shown in Table 2. Finally, the accuracy and applicability of the parameters were verified through tests under different transport conditions.
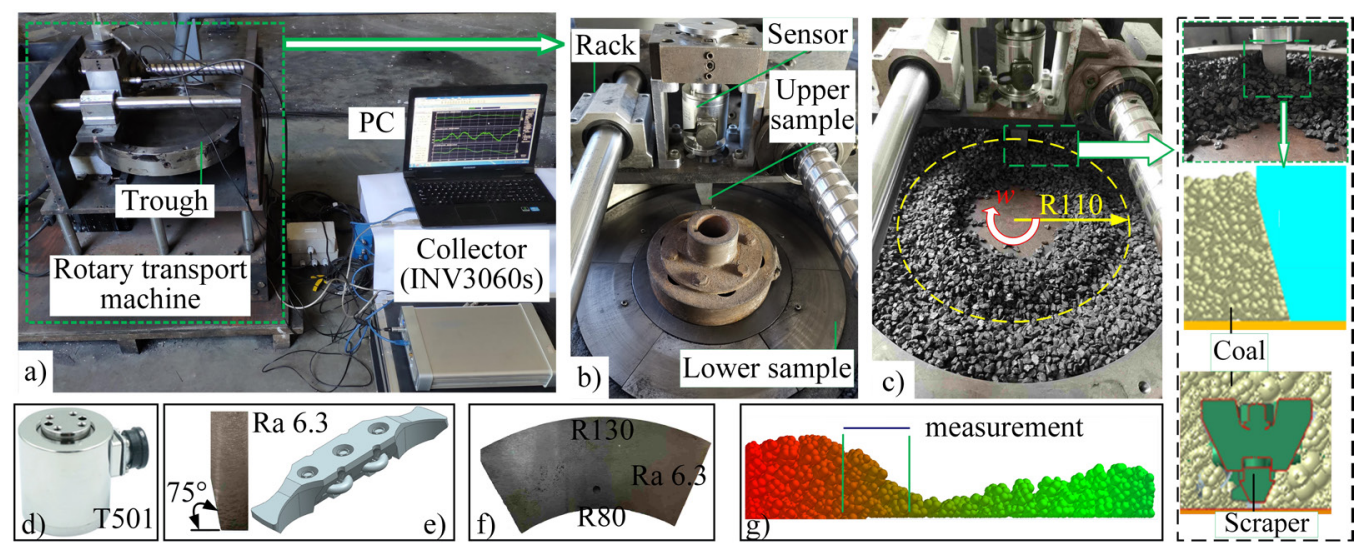

Fig. 3. The test equipment and principle; a) test equipment; b) the rotary transport machine; c) test principle; d) three-dimensional force sensor; e) upper sample; f) lower sample; g) accumulation around the upper sample 


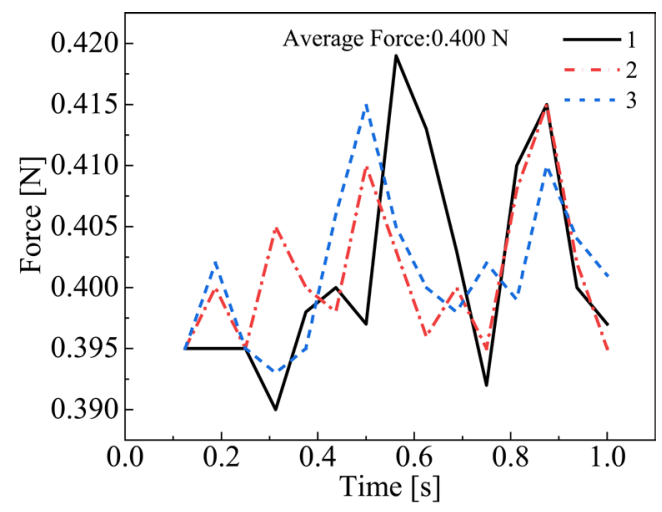

Fig. 4. The force on the upper sample

Table 2. The corrected contact parameters

\begin{tabular}{lcc}
\hline Parameters & Coal-Steel & Coal-Coal \\
\hline Coefficient of restitution & 0.65 & 0.64 \\
\hline Coefficient of static friction & 0.401 & 0.333 \\
\hline Coefficient of rolling friction & 0.032 & 0.041 \\
\hline
\end{tabular}

\subsection{DEM-MBD Coupling Simulation}

\subsubsection{Verification of DEM-MBD Coupling Model}

Due to the short laying length, there is a gap in the speed and tension with the actual situation. The pointby-point method is used to calculate the resistance of long-distance continuous conveying machinery; its main principle is shown in Eq. (1). This research focuses on the fault response of the scraper chain in the chutes, so it only verified the resistance of the loaded side (Points 3 and 4 in Fig. 1) and unloaded side (Points 1 and 2 in Fig. 1), which were calculated based on the parameters of the conveyor to be 348.9 $\mathrm{kN}$ and $35.3 \mathrm{kN}$ respectively [23].

$$
F_{i}=F_{j}+W_{i j},
$$

where $F_{i}[\mathrm{~N}]$ is the tension at the front point, $F_{j}[\mathrm{~N}]$ is the tension at the back point, $W_{i j}[\mathrm{~N}]$ is the running resistance.

The engagement and separation points of the sprockets and the chains are often selected as measuring points, as shown in Fig. 1. The DEM-MBD simulation was carried out under normal conditions to extract the chain tension at four points. According to Eq. (1), the simulation values of the running resistance on the load side and the unloaded side were calculated, and the comparison with the theoretical resistance is listed in Table 3, the relative error under no-load and full-load is less than $8 \%$, which testifies that the simplified model can reflect the mechanical characteristics of scraper conveyor.

Table 3. The comparison between theoretical resistance and tension difference

\begin{tabular}{lcccc}
\hline \multirow{2}{*}{ Items } & \multicolumn{2}{c}{ No-load } & \multicolumn{2}{c}{ Full-load } \\
\cline { 2 - 5 } & $i=4$ & $i=2$ & $i=4$ & $i=2$ \\
& $j=3$ & $j=1$ & $j=3$ & $j=1$ \\
\hline$W_{i j}[\mathrm{kN}]$ & 35.2 & 35.2 & 348.8 & 35.2 \\
\hline$\Delta F_{i j}[\mathrm{kN}]$ & 33.7 & 34.6 & 321.7 & 35.3 \\
\hline Relative error [\%] & 4.26 & 1.70 & 7.77 & 0.28 \\
\hline
\end{tabular}

The chain tension at the middle section of the scraper conveyor provided by the cooperative enterprise (Yaxing Anchor Chain) is about $210 \mathrm{kN}$ to $223 \mathrm{kN}$; the simulation tension is about $200 \mathrm{kN}$, and the maximum relative error between them is $10.3 \%$.

The velocity curve of the chain under fullload condition is shown in Fig. 5, which reveals the fluctuation of "decrease-increase" after the velocity is accelerated. It conforms to the polygon effect [24] and validates the kinematics characteristics of simplified model.

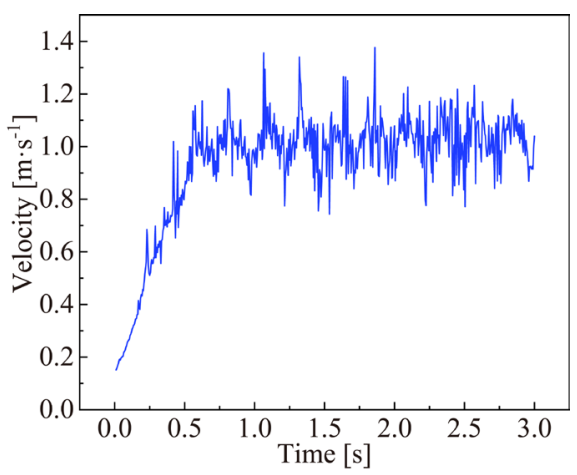

Fig. 5. The velocity of scraper chain

\subsubsection{Design of Fault Conditions}

Chain breaking and chain stuck are the most frequent faults [25]. The small preload, rib spalling of coal wall, and coal blocks stuck at the connection of chutes can cause the chains to jam: we added irregular obstacles in the chute to simulate the chain stuck fault in dynamics software. The increased chain pitch caused by wear, the extreme impact load, the longterm over-carrying and the fatigue crack generated by the synergy of corrosion and other factors can cause the chain to break; we did not deal with the stuck fault in time and set the simulation script for the contact between chain links to simulate chain breaking fault in dynamics software. 


\subsection{Experimental Verification of FEA Model}

\subsubsection{FEA Model of Chain}

The FEA model shown in Fig. 6 was employed to solve the non-linear contact between chain links. The size of the chain link made of $23 \mathrm{MnNiCrMo54}$ (C: $0.22 \%$ ) is $\Phi 34 \mathrm{~mm} \times 126 \mathrm{~mm}$; the properties of the material are listed in Table 4, and the S-N curve is $N S^{2.49}=1.30066 \times 10^{10}$. The entire model was divided into tetrahedral mesh sized $6 \mathrm{~mm}$, yielding 50,997 elements with 76,070 nodes. The frictional contacts were set between the flat chains and the vertical chain, and the coefficient of friction is 0.2 . The right surfaces of flat chain 2 were subjected to boundary loads based on the coupling results, while the left surfaces of flat chain 1 were fixed. The pressure of coal was distributed on vertical chain 1 according to the pressure file derived from EDEM software.

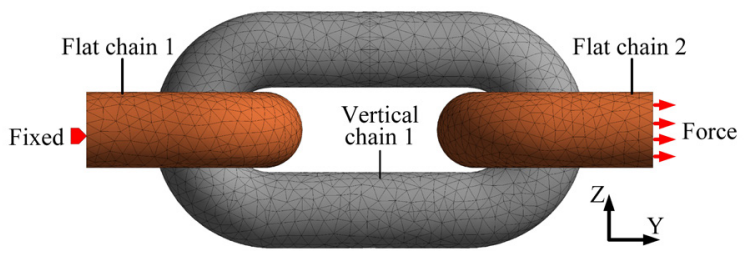

Fig. 6. The FEA model of the chain

Table 4. The mechanical properties of $23 \mathrm{MnNiCrMo54}$

\begin{tabular}{lc}
\hline Properties & Value \\
\hline Density $[\mathrm{kg} \cdot \mathrm{m}-3]$ & 7860 \\
\hline Young's modulus [GPa] & 210 \\
\hline Poisson's ratio & 0.3 \\
\hline Tangent modulus [MPa] & 2444 \\
\hline Yield strength [MPa] & 1166 \\
\hline Tensile yield strength [MPa] & 1254 \\
\hline
\end{tabular}

\subsubsection{Experimental verification}

The tensile testing machine (LAW-5000) was used to obtain the elongation rate of the chain under the test load (1160 kN to $1276 \mathrm{kN})$, as shown in Fig. 7, and the experiment was repeated three times. Based on the model in Fig. 6, the simulation value of the elongation rate is $1.33 \%$. The comparison between the experimental value and the simulated value is shown in Table 5. It can be seen from Table 5 that the relative error does not exceed $5 \%$.

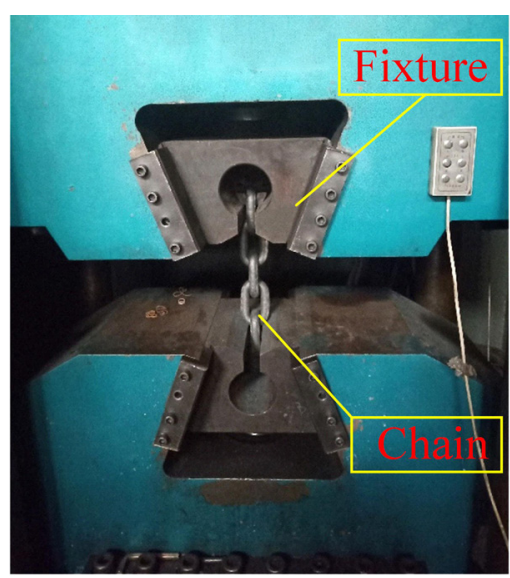

Fig. 7. The microcomputer controlled electro-hydraulic servo tensile testing machine (LAW-5000)

Table 5. The comparison between experimental and simulation values of elongation

\begin{tabular}{|c|c|c|c|c|}
\hline Number & $\begin{array}{c}\text { Experimental } \\
\text { value [\%] }\end{array}$ & $\begin{array}{c}\text { Simulation } \\
\text { value [\%] }\end{array}$ & $\begin{array}{l}\text { Relative } \\
\text { error [\%] }\end{array}$ & $\begin{array}{c}\text { Average } \\
\text { relative } \\
\text { error [\%] }\end{array}$ \\
\hline 1 & 1.40 & \multirow{3}{*}{1.33} & 5.00 & \multirow{3}{*}{4.07} \\
\hline 2 & 1.36 & & 2.20 & \\
\hline 3 & 1.40 & & 5.00 & \\
\hline
\end{tabular}

\section{SIMULATION RESULTS}

When the scraper chains are stuck or broken, the scraper de flects due to the uneven force on both ends, as illustrated in Fig. 8. The surrounding of the deflected scraper is divided into four areas, in which PQ is the stuck side and RS is the deflected side.

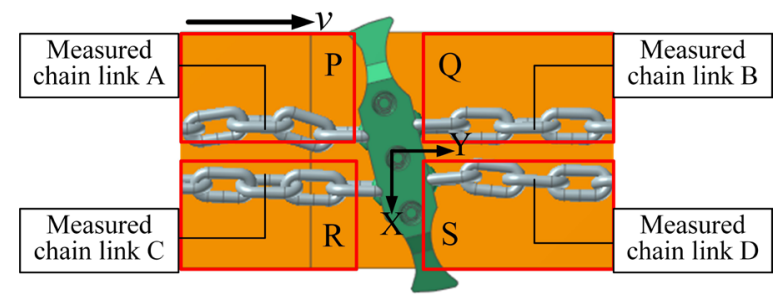

Fig. 8. The areas around the deflected scraper

\subsection{Dynamic Response}

\subsubsection{Operating Tension}

Fig. 9 is the tension diagrams of the measured chain links under faults. When the chain in areas PQ is stuck, the maximum tensions in areas $\mathrm{Q}$ and $\mathrm{R}$ reach 1139.9 $\mathrm{kN}$ and $669.9 \mathrm{kN}$, respectively, and the stuck side is 

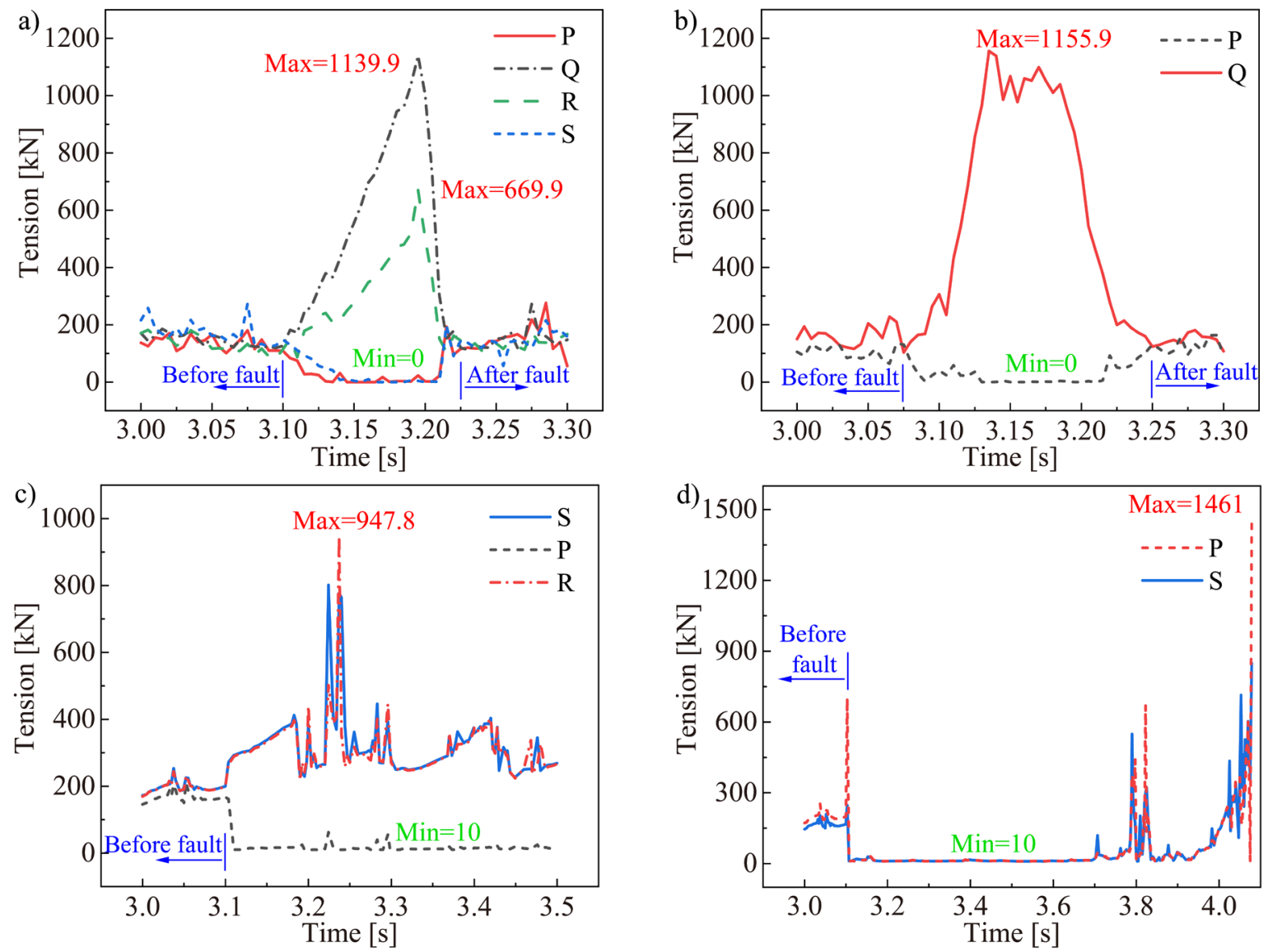

Fig. 9. The tension curves; a) unilateral jamming; b) bilateral jamming; c) unilateral breaking; d) bilateral breaking

larger than the deflected side; the tensions in areas $\mathrm{P}$ and $\mathrm{S}$ are reduced, and the minimums are both $0 \mathrm{kN}$. Furthermore, the tension on the stuck side changes earlier than the deflection side, but both sides return to stability concurrently. The dynamic responses of two chains are identical when both sides of the scraper are stuck, so only the curves on the side PQ are plotted. It can be seen from Fig. 9b that the maximum is 1155.9 $\mathrm{kN}$, and the growth rate of tension is faster. The reason is that when both sides of the scraper are stuck, the scraper cannot deflect, and the growth rate of chain tension cannot be alleviated by deflection.

According to the tension response under chain stuck fault, the areas $\mathrm{Q}$ and $\mathrm{R}$ were set as the fracture points. When the area $\mathrm{Q}$ fails, the tension in area $\mathrm{P}$ decreases and stabilizes at $10 \mathrm{kN}$ to $15 \mathrm{kN}$ due to medium doubled chain; the tension in areas $\mathrm{R}$ and $\mathrm{S}$ increases due to the augment of load, and the maximum is $947.8 \mathrm{kN}$. When the chains in areas Q and $\mathrm{R}$ fracture, the middle scraper deflects instantly, the front and rear scrapers deflect in the opposite direction, and the chains in areas $\mathrm{P}$ and $\mathrm{S}$ are loose, as shown in Fig. 10. With the return of the front and rear scrapers and the deflection of the middle scraper, the chains finally break.

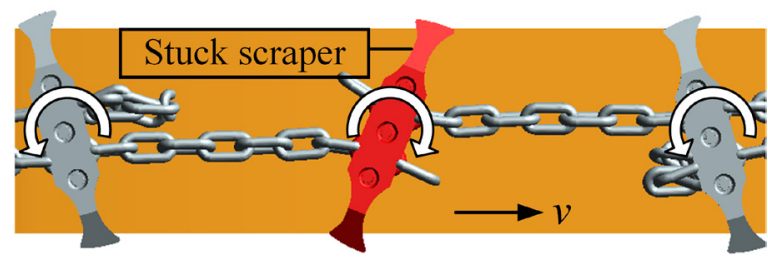

Fig. 10. The deflection of the nearby scrapers

\subsubsection{Velocity of Chains}

Fig. 11 reveals the velocity curves of the measured chain links under faults. In Fig. 11a, compared with the deflected side, the velocity fluctuation of the stuck side is larger, and the two fluctuations in area $\mathrm{P}$ are earlier and later than those in area Q severally. In addition, the chain links on the stuck side have the opposite velocity. The reason is that the chains in areas 

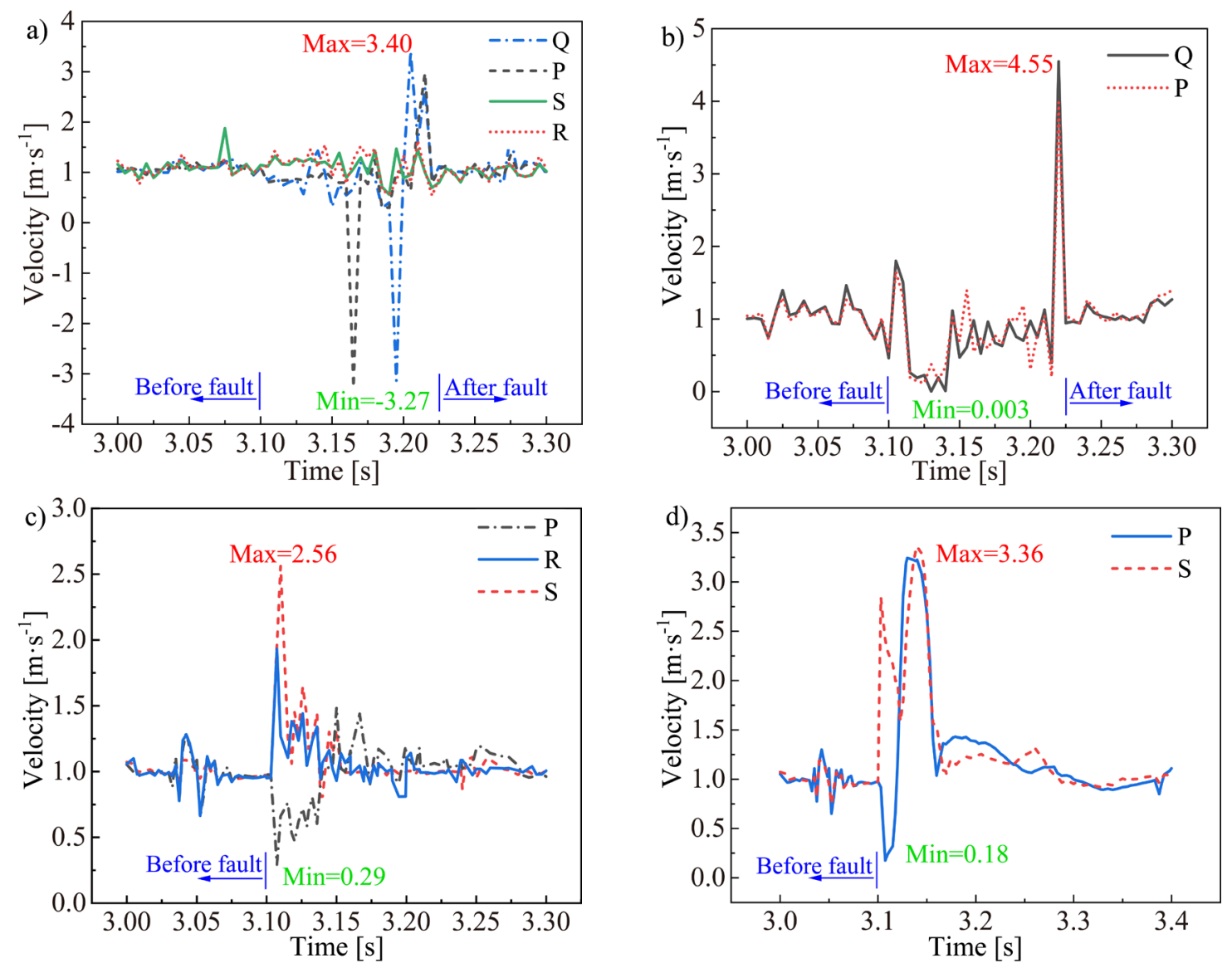

Fig. 11. The velocity curves; a) unilateral jamming; b) bilateral jamming; c) unilateral breaking; d) bilateral breaking

$\mathrm{P}$ and $\mathrm{Q}$ are piled up and tensioned, respectively, and this state is transmitted forward and backward with the stuck scraper as the centre. As a result, the tension at the rear of the tested link along the transportation direction is greater than that at the front in a short time. The velocity at the front and rear of the scraper are synchronous when two sides are stuck. The lowest velocity is almost $0 \mathrm{~m} \cdot \mathrm{s}^{-1}$, and there is a large positive mutation before recovery.

When the chain in area Q breaks, the velocity in area $\mathrm{P}$ is reduced by the deflection and impact of the scraper, and the velocities in areas $\mathrm{R}$ and $\mathrm{S}$ increase with different amplitudes. When the chains at areas $\mathrm{Q}$ and $\mathrm{R}$ both break, the velocity in area P fluctuates from decrease to increase, and the velocity in area $\mathrm{S}$ increases.

\subsubsection{Acceleration in the Z-Direction}

Fig. 12 shows the Z-direction acceleration curves of the measured chain links under faults. The scraper vibration can affect the chain vibration under normal conditions, and their vibrations are transmitted through the chain tension. From the chain tension curves under fault conditions (Fig. 9), it can be seen that when the PQ side of the scraper is stuck, the stuck scraper vibrates and deflects due to the external load. The scraper deflection loosens the chains in areas $\mathrm{P}$ and $\mathrm{S}$, blocks the transmission of chain tension, causes the chain vibration in this area to weaken, and the minimum can reach 0 . Additionally, the scraper deflection causes the chains in areas $\mathrm{Q}$ and $\mathrm{R}$ to be tightened, and the chain tension and its transmittance increases, resulting in the increase of chain vibration, up to $33 \mathrm{~m} \cdot \mathrm{s}^{-2}$. Since the chain vibrations in areas $\mathrm{R}$ and $\mathrm{S}$ are the same as those in areas $\mathrm{Q}$ and $\mathrm{P}$, respectively, they are not repeated in the figure. The results of bilateral jamming and unilateral fracture of the chain are the same as above.

When the chains in areas $\mathrm{Q}$ and $\mathrm{R}$ break, the middle scraper deflects quickly, and then the front and rear scrapers deflect in opposite directions, as shown in Fig. 10. The remaining chains are piled up, the 

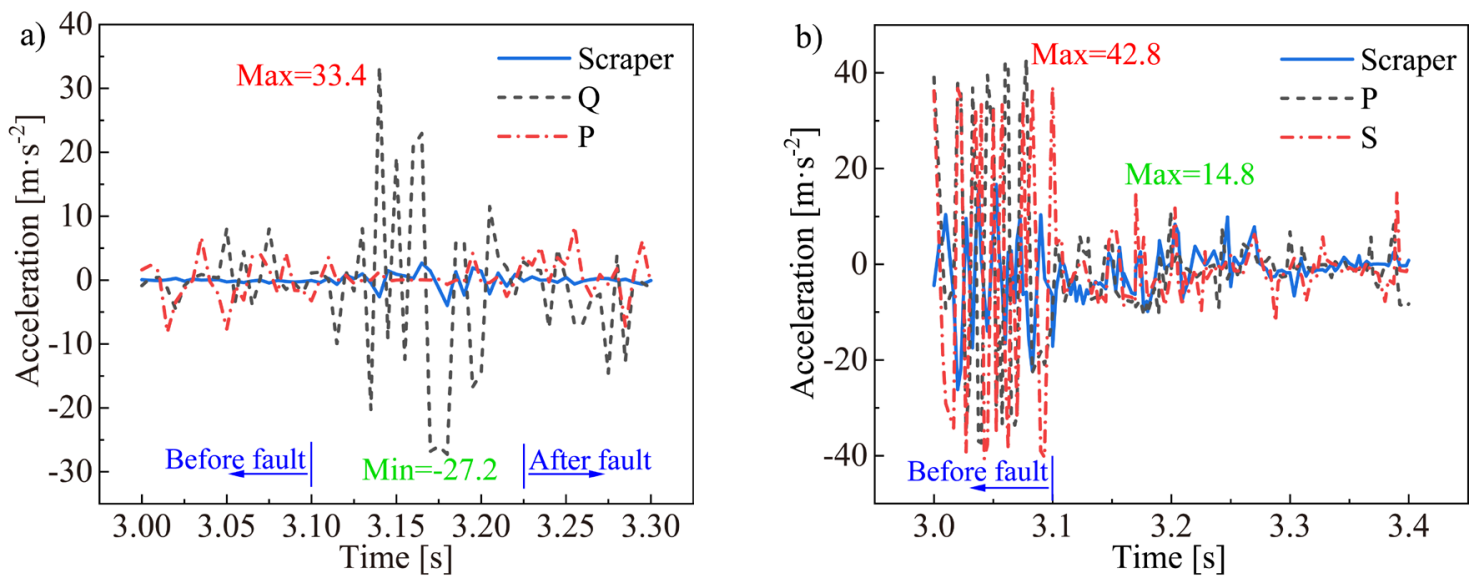

Fig. 12. The curves of acceleration in the Z-direction; a) unilateral jamming; and b) bilateral breaking

tension and its transmittance are reduced, resulting in reduced vibration of the chain and scraper.

\subsection{Discrete Characteristics of Bulk Coal}

\subsubsection{Velocity of Bulk Coal}

The velocity of bulk coal under unilateral jamming is shown in Fig. 13.

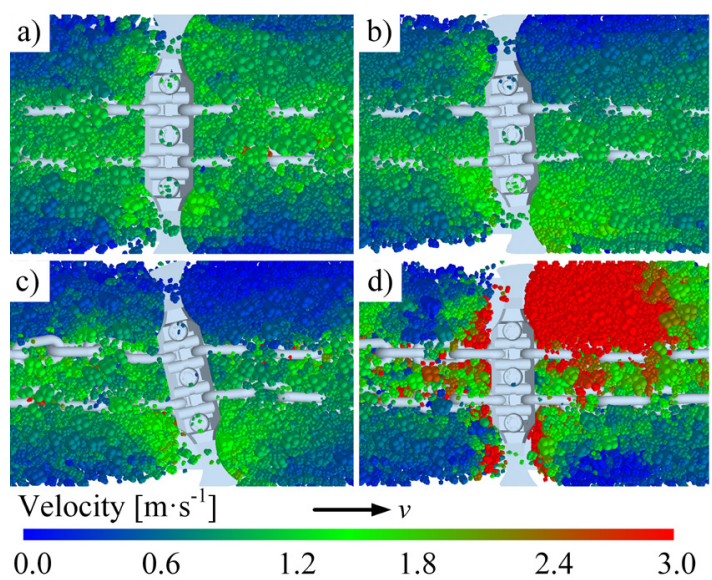

Fig. 13. The velocity cloud diagram of bulk coal under unilateral jamming; a) before fault; b) initial stage of fault;

c) middle stage of fault; $d$ ) after fault

The farther the bulk coal is away from the scraper and chains, the lower the velocity. When the scraper starts to deflect, the velocity of the bulk coal in area $\mathrm{Q}$ decreases first; the velocity of the bulk coal in area $\mathrm{S}$ increases briefly. During the continuous deflection of the scraper to the maximum, the bulk coal in areas $\mathrm{P}$ and $\mathrm{Q}$ continue to accumulate, and the bulk coal in area $\mathrm{S}$ gradually return to the normal velocity. As the stuck side lags behind the deflected side by an angle, the velocity of the bulk coal in area Q instantly increases when the scraper returns to be steady, which can reach a maximum of $10.15 \mathrm{~m} \cdot \mathrm{s}^{-1}$. The flying bulk coal may injure the staff. The result when both sides are stuck is the same as the result on the stuck side in Fig. 13.

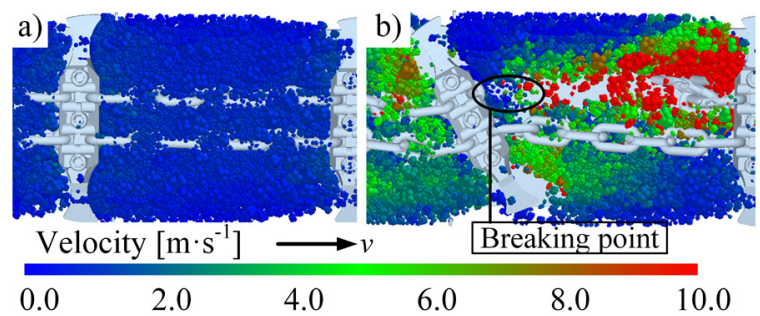

Fig. 14. The velocity cloud diagram of bulk coal under unilateral breaking; a) before fault; and b) after fault

\subsubsection{Wear of Medium Plate}

A layer of coal with a smaller size is often covered between the medium plate and scraper chain. When the scraper chain moves, three-body abrasive wear is formed [13], as shown in Fig. 15. Due to the short simulation time, only the qualitative analysis and prediction of the wear area of the medium plate was carried out; the material of the medium plate is NM450 (C: $\leq 0.35 \%)$. The wear depth in the EDEM software was calculated by Eq. (2).

$$
h=Q / A=W F_{n} d_{t} / A,
$$

where $Q\left[\mathrm{~m}^{3}\right]$ is the volume of material removed, $W$ $\left[\mathrm{Pa}^{-1}\right]$ is the wear constant, the size is $1.2 \times 10^{-12} \mathrm{~Pa}^{-1}$, $F_{n}[\mathrm{~N}]$ is the normal force, $d_{t}[\mathrm{~m}]$ is the tangential distance moved, and $A\left[\mathrm{~m}^{2}\right]$ is the area of material removed. 


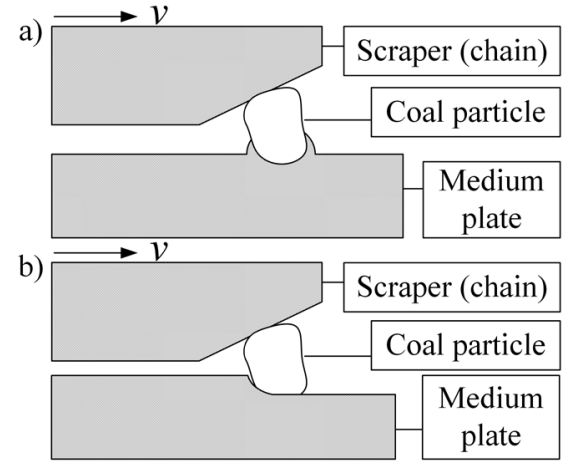

Fig. 15. The three-body abrasive wear; a) furrow effect; b) micro-cutting effect

It can be seen from Fig. 16 that the wear area of the medium plate is concentrated near the chain paths, which is consistent with the wear area of the scrapped medium plate. The unilateral jamming condition deepens the wear at the chain paths. In addition to deepening the wear at the chain paths, the bilateral jamming condition also form new wear areas at the centre and both ends of scraper. When the chain fractures, curved wear marks are generated on the medium plate due to the severe deflection of scraper.

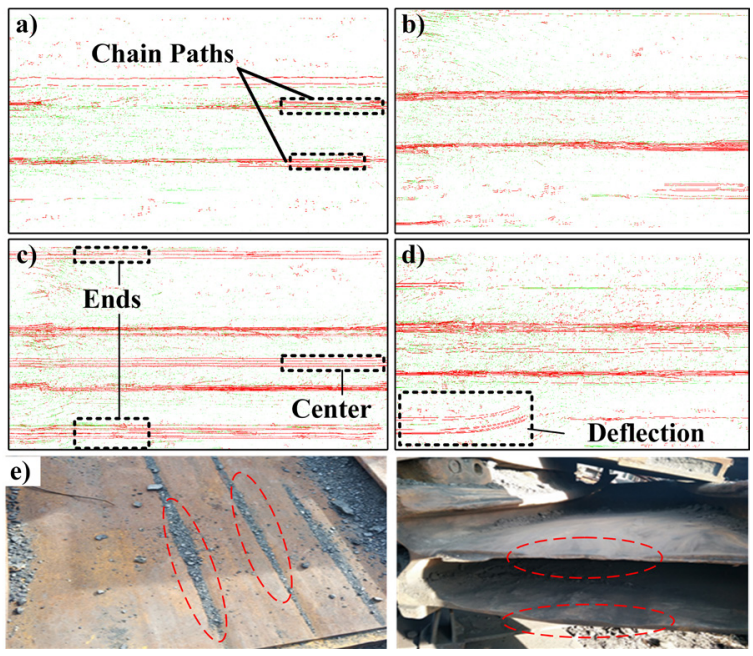

Fig. 16. The wear areas of medium plate;

a) normal; b) unilateral jamming; c) bilateral jamming;

d) chain breaking; e) the actual wear area

\subsection{Results of FEA}

\subsubsection{Static Structural Analysis}

The results of static analysis under normal load and stuck fault load were obtained by applying tension of $180 \mathrm{kN}$ and $1155.9 \mathrm{kN}$ to the FEA model, shown in Figs. 17 and 18. The maximum equivalent stress occurs at the inner side of the connection between the curved section and the straight section. The equivalent stress and elastic strain under normal load are less than the yield strength. The maximum equivalent stress under fault load is $1238.2 \mathrm{MPa}$, which is higher than the yield strength, and the maximum plastic strain is 0.10098. The above results explain that the chain has a high degree of damage when the chain is stuck. To prevent chain breaking accident, the damaged chain should be replaced in time.

a) Equivalent Stress/MPa

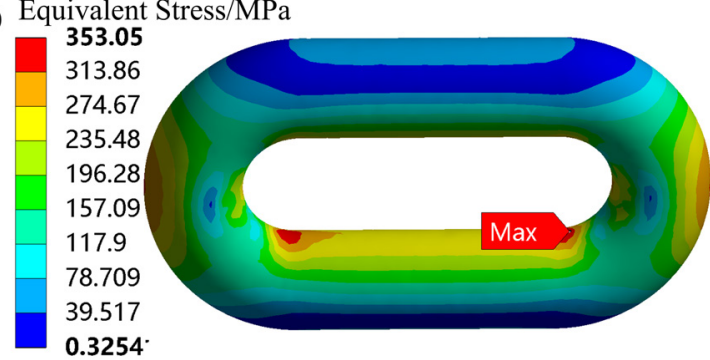

b)

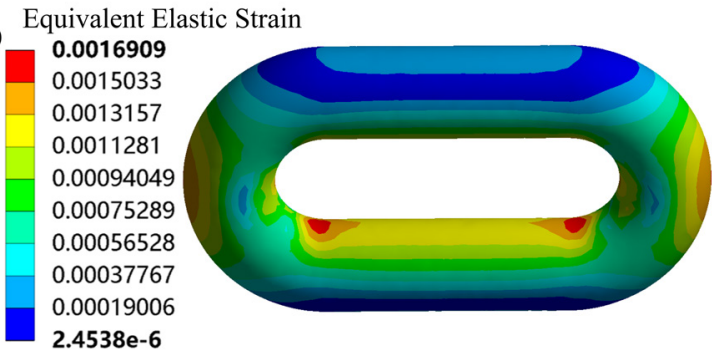

Fig. 17. The results of statics analysis under normal load; a) equivalent stress, b) equivalent elastic strain

a) Equivalent Stress/MPa

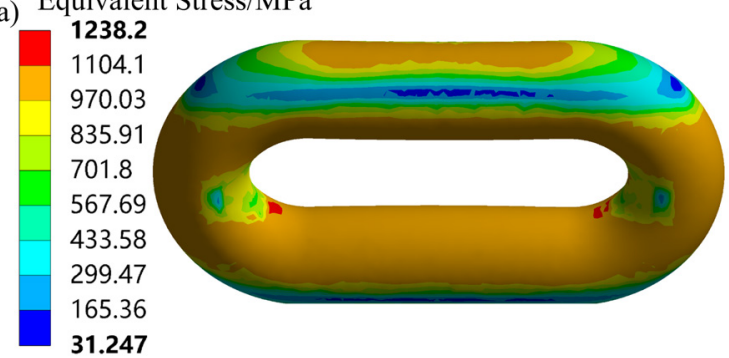

b) Equivalent Plastic Strain

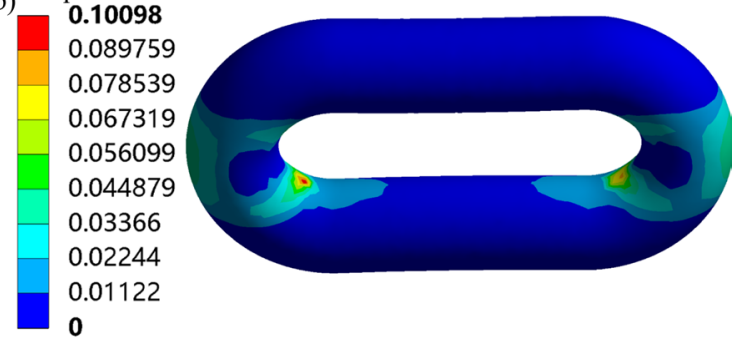

Fig. 18. The results of static analysis under stuck fault load; a) equivalent stress, b) equivalent elastic strain 
Based on the equipment in Fig. 7, the deformation results of the chain were verified. The comparison of elongation is shown in Table 6. It can be seen that the relative error between simulation and experiment does not exceed $4 \%$.

Table 6. The comparison between experimental and simulation values of elongation

\begin{tabular}{lccccc}
\hline Number & \multicolumn{2}{c}{$\begin{array}{c}\text { Experimental value } \\
{[\%]}\end{array}$} & $\begin{array}{c}\text { Simulation } \\
\text { value [\%] }\end{array}$ & $\begin{array}{c}\text { Average relative } \\
\text { error [\%] }\end{array}$ \\
\hline Normal load & 0.220 & 0.225 & 0.223 & 0.215 & 3.43 \\
\hline Fault load & 1.27 & 1.25 & 1.28 & 1.22 & 3.68 \\
\hline
\end{tabular}

\subsubsection{Fatigue Life}

According to the coupling results, the load history for one cycle under normal conditions was constructed, shown in Fig. 19. The fatigue life was calculated based on the results of static analysis and linear cumulative damage theory. The theory can be expressed as:

$$
D=\sum_{1}^{m}\left(n_{i} / N_{i}\right)
$$

where $D$ is the total damage, $n_{i}$ is the number of cycles under stress level, $N_{i}$ is the life under stress level, $m$ is the number of stresses.

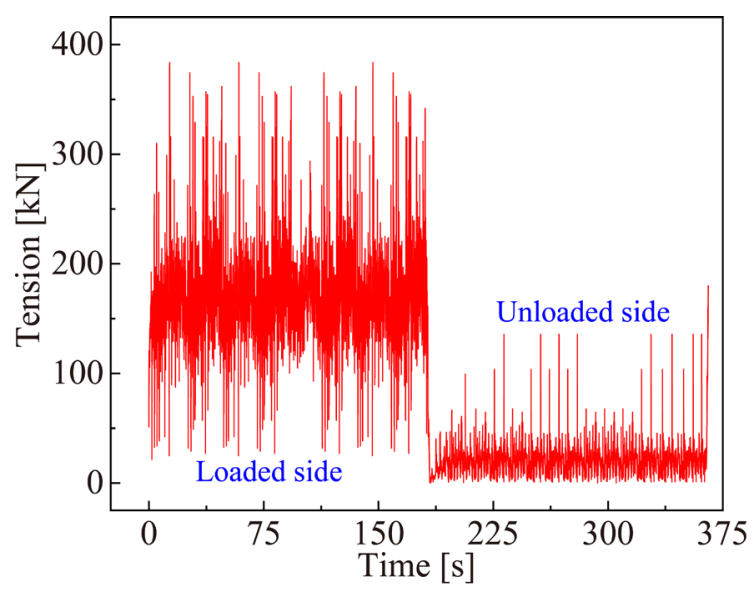

Fig. 19. The load history under normal condition

Based on Fig. 19, the stuck fault load was added to obtain the fatigue life. The fatigue life is displayed in Fig. 20. The lowest parts of the life under the two conditions are the contact position and the inner side of the connection, and the lowest values are 20,070 and 383.8 cycles respectively. The life under normal load is 52 times that under fault load. The chain near the stuck position should be overhauled and replaced in time to prevent the chain from breaking. a)

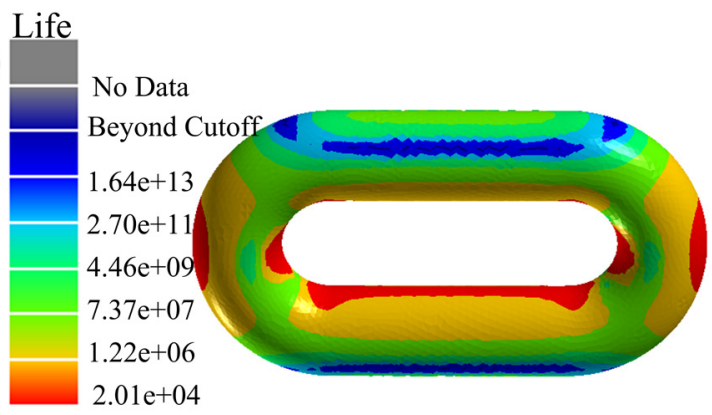

b)

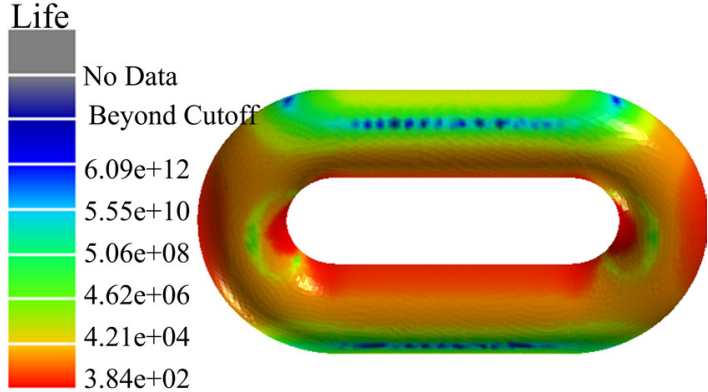

Fig. 20. The fatigue life; a) normal load, b) fault load

\section{ANALYSIS OF FACTORS AFFECTING CRACK GROWTH}

\subsection{Plackett-Burman Test}

\subsubsection{Design of Test}

Defects in structure or material are inevitable, of which cracking is the most common. According to load and propagation form of the cracks, they can be divided into three types: open type, slip type, and tear type, as shown in Fig. 21.

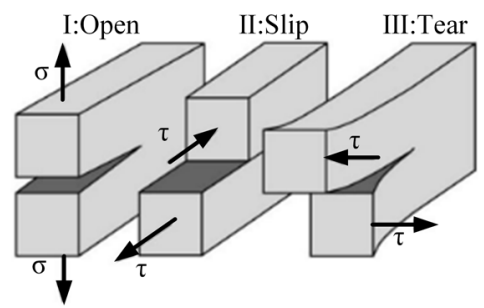

Fig. 21. The types of the crack

Stress intensity factor (SIF) $K$ is defined as the strength of the stress field around the crack front of linear elastic material. The main stresses in the chain link are tensile and compressive stresses. The fracture type of chain link is Type-I, which SIF $K_{1}$ is expressed as:

$$
K_{1}=\sigma \sqrt{\pi a} f(a, W),
$$


where $\sigma[\mathrm{MPa}]$ is the normal stress, $a[\mathrm{~mm}]$ is crack size, $W[\mathrm{~mm}]$ is the size of the component in the direction of crack depth, $f(a, W)$ is the shape correction function.

The Plackett-Burman test determines the significance of the factor by comparing the response variables at different levels of each factor [26]. Based on the results of FEA, a semi-elliptical crack was set on the inner side of the connection. Through presimulation, it is found that the positive or negative initial angle of the crack in Fig. 22 does not affect the result. Therefore, the crack depth, depth-length ratio, initial angle and tensile load were taken as factors, and the maximum $K_{1 \max }$ of the type-I SIF at the crack front was used as the response variable to carry out the screening test. Each factor was set to high and low levels, as listed in Table 7, where the virtual parameters are used for error estimation.

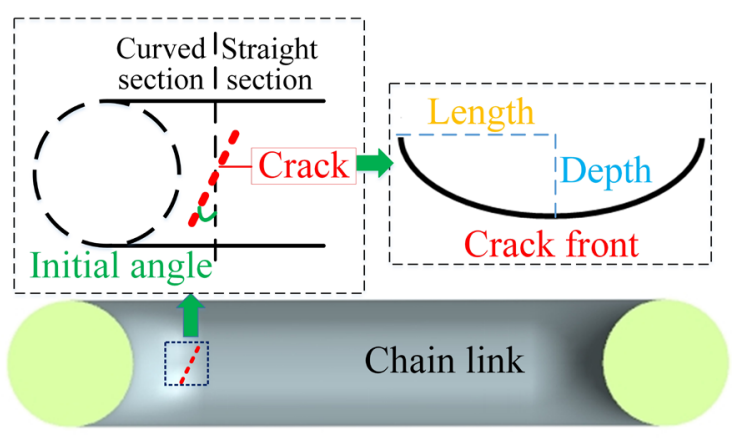

Fig. 22. The factors of crack

Table 7. The factors and levels of Plackett-Burman test

\begin{tabular}{lcc}
\hline Parameters & Low $(-1)$ & High $(+1)$ \\
\hline Crack depth $L[\mathrm{~mm}]$ & 0.2 & 1 \\
\hline Depth to length ratio $B$ & 0.2 & 0.8 \\
\hline Initial angle $A\left[^{\circ}\right]$ & 0 & 60 \\
\hline Tensile load $F[\mathrm{kN}]$ & 90 & 270 \\
\hline Virtual parameters & -1 & +1 \\
\hline
\end{tabular}

\subsubsection{Results of Testing}

Table 8 demonstrates the plan and results of test, and the significance ranking of factors on $K_{1 \max }$ is listed in Fig. 23. From the pareto chart, the tensile load, initial angle, and crack depth are significant on $K_{1 \max }(t>2.36462)$, and the depth to length ratio is not significant. The tensile load and crack depth are positive effects, and the initial angle is negative. The order of significance is $F>A>L$. Therefore, the significant factors were selected for the following tests.
Table 8. The plan and result of the Plackett-Burman test

\begin{tabular}{cccccc}
\hline Number & $L$ & $B$ & $A$ & $F$ & $K_{1 \max }\left[\mathrm{MPa} \cdot \mathrm{mm}^{-1 / 2}\right]$ \\
\hline 1 & 1 & 1 & -1 & 1 & 682.48 \\
\hline 2 & -1 & 1 & 1 & -1 & 31.16 \\
\hline 3 & 1 & -1 & 1 & 1 & 299.59 \\
\hline 4 & -1 & 1 & -1 & 1 & 309.75 \\
\hline 5 & -1 & -1 & 1 & -1 & 43.38 \\
\hline 6 & -1 & -1 & -1 & 1 & 424.65 \\
\hline 7 & 1 & -1 & -1 & -1 & 293.58 \\
\hline 8 & 1 & 1 & -1 & -1 & 226.25 \\
\hline 9 & 1 & 1 & 1 & -1 & 71.88 \\
\hline 10 & -1 & 1 & 1 & 1 & 94.07 \\
\hline 11 & 1 & -1 & 1 & 1 & 299.59 \\
\hline 12 & -1 & -1 & -1 & -1 & 140.78
\end{tabular}

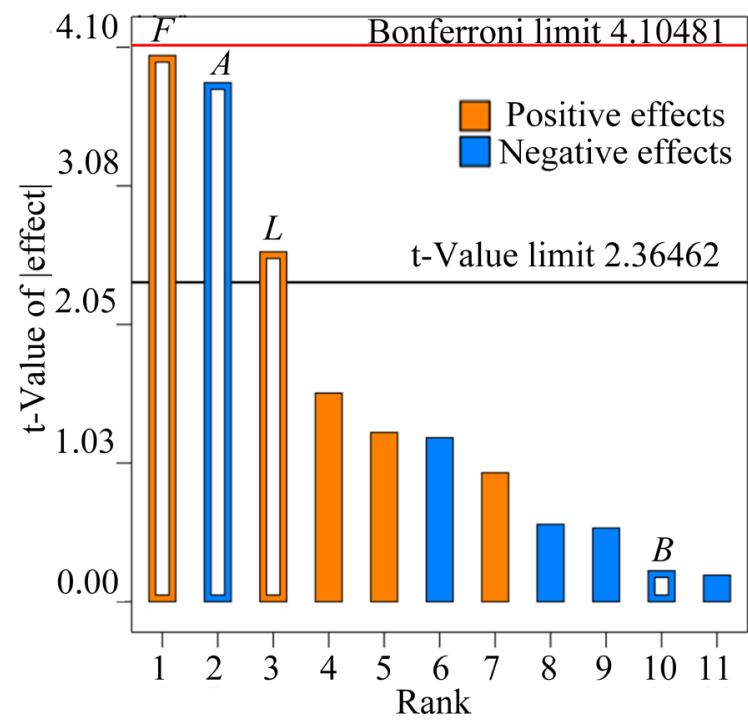

Fig. 23. The pareto chart

\subsection{Single Factor Test}

The design of the single factor test is shown in Table 9, and the depth to length ratio is fixed at 0.5 . The $K_{1}$ on the crack front is plotted as Fig. 24, where the ratio of the arc length from a point to the end of crack front to the total arc length is the relative position of the point. In Fig. 24, $K_{1}$ aggrandizes with the increase of crack depth, and the growth rate gradually slows down. $K_{1}$ is negatively correlated with the initial angle; the larger the initial angle, the greater the decrease in $K_{1}$, and the smoother the curve of $K_{1} . K_{1}$ is positively correlated with the tensile load, and the increase of $K_{1}$ on the crack front is almost constant; the lower the load, the smoother the curve. In addition, as the initial angle increases, the maximum position of $K_{1}$ moves to the end of crack front near the load side. 
Table 9. The design of the single factor test

\begin{tabular}{lccc}
\hline Number & $L[\mathrm{~mm}]$ & $A\left[^{\circ}\right]$ & $F[\mathrm{kN}]$ \\
\hline 1 & 0.2 to 1.0 & 0 & 180 \\
\hline 2 & 0.6 & 0 to 60 & 180 \\
\hline 3 & 0.6 & 0 & 90 to 270 \\
\hline
\end{tabular}
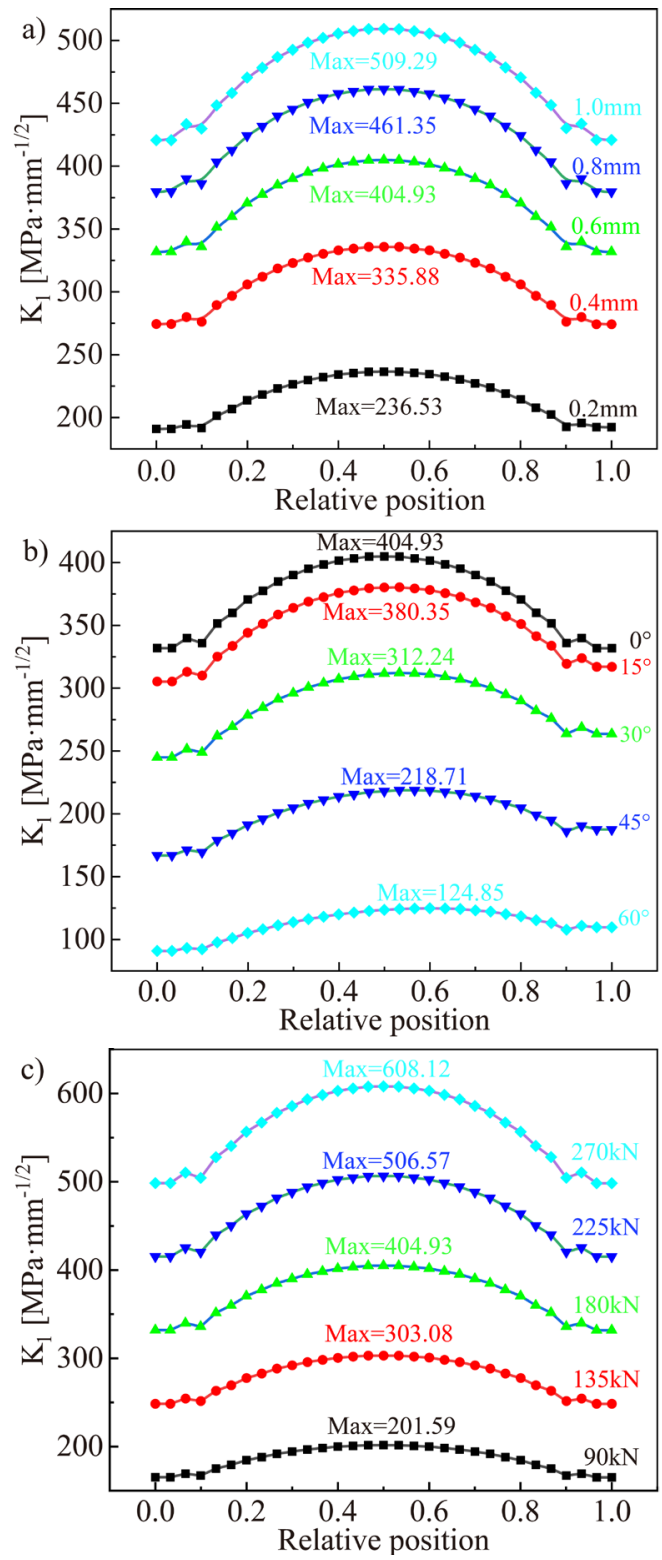

Fig. 24. The effects on $K_{1}$;

a) crack depth; b) initial angle; c) tensile load

\subsection{Box-Behnken Test}

\subsubsection{Design of Test}

Based on the results of the Plackett-Burman test, the Box-Behnken test was designed with the $K_{1 \max }$ as the response value. Each factor was set to high $(+1)$, medium $(0)$, and low $(-1)$ three levels, as listed in Table 10. The entire design includes 12 sets of factorial tests and three sets of central tests for error estimation.

Table 10. The factors and levels of Box-Behnken test

\begin{tabular}{lccc}
\hline Factors & -1 & 0 & +1 \\
\hline$L[\mathrm{~mm}]$ & 0.2 & 0.6 & 1 \\
\hline$A\left[^{\circ}\right]$ & 0 & 30 & 60 \\
\hline$F[\mathrm{kN}]$ & 90 & 180 & 270 \\
\hline
\end{tabular}

\subsubsection{Results of Test}

Based on the results of the test in Table 11, the quadratic regression polynomial between factors and $K_{1 \max }$ was constructed by Design-Expert software as:

$$
\begin{aligned}
K_{1 \max } & =-124.6+245.42 L+5.49 A+1.5 F-3.82 L A \\
& +1.46 L F-0.026 A F-126.34 L^{2}-0.05 A^{2} \\
& -0.00017 F^{2} .
\end{aligned}
$$

\begin{tabular}{|c|c|c|c|c|}
\hline Number & $L$ & $A$ & $F$ & $K_{1 \max }\left[\mathrm{MPa} \cdot \mathrm{mm}^{-1 / 2}\right]$ \\
\hline 1 & -1 & -1 & 0 & 239.98 \\
\hline 2 & 1 & -1 & 0 & 509.29 \\
\hline 3 & -1 & 1 & 0 & 72.71 \\
\hline 4 & 1 & 1 & 0 & 158.86 \\
\hline 5 & -1 & 0 & -1 & 92.02 \\
\hline 6 & 1 & 0 & -1 & 196.12 \\
\hline 7 & -1 & 0 & 1 & 277.36 \\
\hline 8 & 1 & 0 & 1 & 591.39 \\
\hline 9 & 0 & -1 & -1 & 201.01 \\
\hline 10 & 0 & 1 & -1 & 62.137 \\
\hline 11 & 0 & -1 & 1 & 605.63 \\
\hline 12 & 0 & 1 & 1 & 187.59 \\
\hline 13 & 0 & 0 & 0 & 310.23 \\
\hline 14 & 0 & 0 & 0 & 305.57 \\
\hline 15 & 0 & 0 & 0 & 316.51 \\
\hline
\end{tabular}

Table 11. The results of the Box-Behnken test

The variance analysis of the regression polynomial is listed in Table 12. The model is extremely prominent $(P<0.0001)$, and the lack of fit is not prominent $(P=0.0839>0.05)$. The coefficient of variation $(\mathrm{CV})$ is small $(5.3 \%)$, the $R$ Square before $(0.998)$ and after $(0.993)$ correction is close to 1 , and the accuracy is high $(46>4)$. It can be concluded that the constructed quadratic model has high fitting accuracy and can characterize the relationship between $K_{1 \max }$ and factors. The significance of term 
was acquired by F-test. The larger the F value, the more prominent the impact. When the significance level is 0.05 , the significances of terms are ranked as: $F>A>L>A F>L F>L A>A^{2}>L^{2}$.

Table 12. The variance analysis of polynomial

\begin{tabular}{lccc}
\hline Source & Sum of Squares & $F$-value & $P$-value \\
\hline Model & $4.21 \mathrm{E}+05$ & 220.05 & $<0.0001$ \\
\hline$L$ & 74805.19 & 351.97 & $<0.0001$ \\
\hline$A$ & $1.44 \mathrm{E}+05$ & 679.18 & $<0.0001$ \\
\hline$F$ & $1.54 \mathrm{E}+05$ & 725.54 & $<0.0001$ \\
\hline$L A$ & 8386.9 & 39.46 & 0.0015 \\
\hline$L F$ & 11017.65 & 51.84 & 0.0008 \\
\hline$A F$ & 19483.55 & 91.67 & 0.0002 \\
\hline$L^{2}$ & 1508.79 & 7.1 & 0.0446 \\
\hline$A^{2}$ & 7592.13 & 35.72 & 0.0019 \\
\hline$F 2$ & 6.56 & 0.0309 & 0.8674 \\
\hline Lack of Fit & 1002.4 & 11.09 & 0.0839 \\
\hline$R^{2}=0.998 ;$ & $R_{a}^{2}=0.993 ;$ & $C V=5.3 \% ;$ & Precision $=46$ \\
\hline
\end{tabular}

\subsubsection{The Effect of Interaction}

Through the three-dimensional response surface graph, the relationship between the interaction and the response value can be understood intuitively. When a factor is at 0 level, the variation of $K_{1 \max }$ with the interaction is shown in Fig. 25. It can be seen that the crack depth and tensile load are positively related to the rate of change of $K_{1 \max }$ with other factors, while the initial angle has a negative effect on the rate of change of $K_{1 \max }$ with other factors.

\section{CONCLUSIONS}

In this paper, a fault simulation method of scraper conveyor based on MBD-DEM-FEM coupling was used. The DEM-MBD-FEM models were established and verified by experiments and theory, the response data of the chain and bulk coal under chain stuck and chain-breaking faults were obtained, and some results were verified. For the fracture failure, the single factor test and response surface test were used to investigate the single and interactive effects of factors on the SIF.

1. With the force and stacking angle as response values, a rotary transport testing machine was designed to modify the contact parameters of coal. The DEM-MBD coupling model was verified, and the relative error between tension difference and resistance is less than $8 \%$. The static structure model of the chain was verified
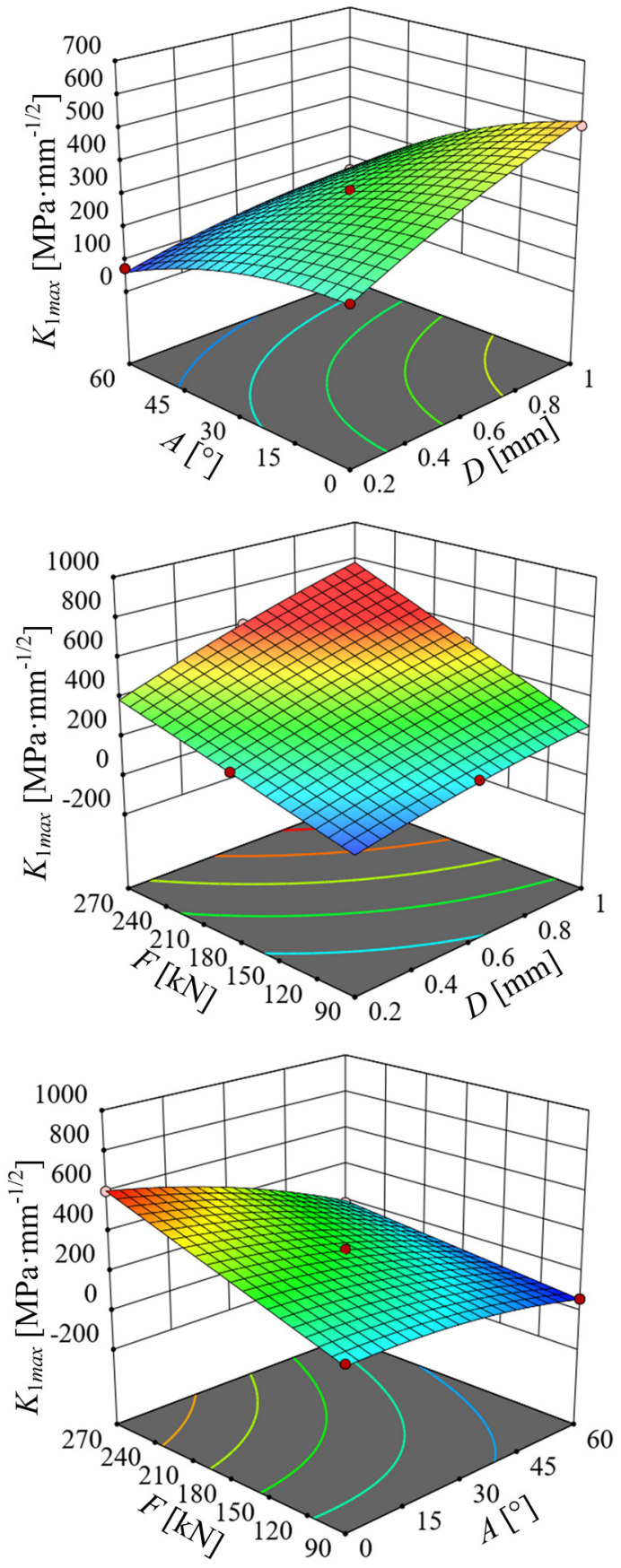

Fig. 25. Response surface of interaction to $K_{1 \text { max }}$; a) $D A$; b) $D F$; c) $A F$

by the chain tensile experiment, and the relative error is less than $5 \%$.

2. In chain jamming and breaking faults, the deflection of scraper causes the uneven stress on chains. The tension of the tensioned chain increases rapidly, resulting in the torsion and vibration of chains. The tension, velocity, and 
vibration of the loose chain decrease. The fault load leads to the plastic deformation of chain link and reduces its fatigue life. The wear of the medium plate is concentrated on the chain paths, which is consistent with the actual situation. In addition, the chain faults cause new wear on the centre and both sides of the medium plate surface.

3. The tensile load, initial angle and crack depth are significant on $K_{1}$. Through response surface analysis, both the crack depth and tensile load are positively correlated with $K_{1}$, and the initial angle is negatively correlated with $K_{1}$. The initial angle has a negative effect on the change rate of $K_{1 \max }$ with other factors, while the crack depth and tensile load positively affect it.

\section{ACKNOWLEDGEMENTS}

This project was supported by National Natural Science Foundation of China (Grant Nos. 51875386 and 51804207) and the Fund for Shanxi "1331" Project.

\section{REFERENCES}

[1] Zhang, X., Li, W., Zhu, Z., Yang, S., Jiang, F. (2019). Fault detection for the scraper chain based on vibration analysis using the adaptive optimal kernel time-frequency representation. Shock and Vibration, vol. 2019, art. ID 6986240, DOI:10.1155/2019/6986240.

[2] Thirumurugan, R., Gnanasekar, N. (2020). Influence of finite element model, load-sharing and load distribution on crack propagation path in spur gear drive. Engineering Failure Analysis, vol. 110, art. ID 104383, D0l:10.1016/j. engfailanal.2020.104383.

[3] Li, J., Ma, Q. (2018). Mechanical characteristics of the scraper chain transmission system under the condition of chain blocked. Journal of the China Coal Society, vol. 43, p. 591599, D0I:10.13225/j.cnki.jccs.2018.0384. (in Chinese)

[4] Jiang, S.B., Zeng, Q.L., Wang, G., Gao, K.D., Wang, Q.Y., Hidenori, K. (2018). Contact analysis of chain drive in scraper conveyor based on dynamic meshing properties. International Journal of Simulation Modelling, vol. 17, no. 1, p. 81-91, DOl:10.2507/ijsimm17(1)418.

[5] Wang, D.G., Zhang, J., Zhu, Z.C., Gang, S., Xiang, L. (2019). Crack initiation characteristics of ring chain of heavy-duty scraper conveyor under time-varying loads. Advances in Mechanical Engineering, vol. 11, no. 9, p. 11, DOI:10.1177/1687814019880366.

[6] Wang, X.W., Li, B., Wang, S.W., Yang, Z.J., Cai, L. (2018). The transporting efficiency and mechanical behavior analysis of scraper conveyor. Proceedings of the Institution of Mechanical Engineers Part C-Journal of Mechanical Engineering Science, vol. 232, no. 18, p. 3315-3324, Dol:10.1177/0954406217734002.
[7] Jiang, S.B., Ren, W.J., Mao, Q.H., Zeng, Q.L., Yu, P.F., Gao, K.D., Wang, L. (2021). Dynamic analysis of the scraper conveyor under abnormal operating conditions based on the vibration and speed characteristics. Shock and Vibration, vol. 2021, art. ID 8887744, D0I:10.1155/2021/8887744.

[8] Xie, C., Liu, Z., Mao, J., Xie, M., Lu, J. (2018). Analysis of torsional vibration characteristics of scraper conveyor on chain blocked condition. Journal of the China Coal Society, vol. 43, no. 8, p. 2348-2354, D0l:10.13225/j.cnki.jccs.2017.1722. (in Chinese)

[9] Wang, X.W., Wang, S.P., Long, R.S., Yang, Z.J., Liu, G.P. (2016). Rigid-flexible coupled dynamic contact analysis for a chains drive system of a heavy scraper conveyer during loading startup. Journal of Vibration and Shock, vol. 35, no. 11, p. 34-40, Dol:10.13465/j.cnki.jvs.2016.11.006. (in Chinese)

[10] Jiang, S.B., Zhang, X., Gao, K.D., Gao, J., Wang, Q.Y., Hidenori, K. (2017). Multi-body dynamics and vibration analysis of chain assembly in armoured face conveyor. International Journal of Simulation Modelling, vol. 16, no. 3, p. 458-470, D0l:10.2507/ ijsimm16(3)8.391.

[11] Dong, Y.S., Si, F.Q., Cao, Y., Jin, W., Ren, S.J. (2021). A new mechanistic model for abrasive erosion using discrete element method. Powder Technology, vol. 380, p. 486-496, D0I:10.1016/j.powtec.2020.11.017.

[12] Wang, X.W., Li, B., Yang, Z.J. (2018). Analysis of the bulk coal transport state of a scraper conveyor using the discrete element method. Strojniški vestnik - Journal of Mechanical Engineering, vol. 64, no. 1, p. 37-46, D0l:10.5545/svjme.2017.4790.

[13] Xia, R., Wang, X., Li, B., Wei, X., Yang, Z. (2019). Discrete element method-(DEM-) based study on the wear mechanism and wear regularity in scraper conveyor chutes. Mathematical Problems in Engineering, vol. 2019, art. ID 4191570, DOI:10.1155/2019/4191570.

[14] Xia, R., Wang, X., Li, B., Wei, X., Yang, Z. (2019). The prediction of wear on a scraper conveyor chute affected by different factors based on the discrete element method. Proceedings of the Institution of Mechanical Engineers, Part C: Journal of Mechanical Engineering Science, vol. 233, no. 17, p. 62296239, DOI:10.1177/0954406219861130.

[15] Xia, R., Li, B., Wang, X.W., Li, T.J., Yang, Z.J. (2019). Measurement and calibration of the discrete element parameters of wet bulk coal. Measurement, vol. 142, p. 8495, D0I:10.1016/j.measurement.2019.04.069.

[16] Li, T.J., Wang, X.W., Li, B., Li, J.L., Yang, Z.J. (2018). Optimization method for coal particle model parameters based on discrete element method. China Powder Science and Technology, vol. 24, no. 05, p. 6-12, Dol:10.13732/j. issn.1008-5548.2018.05.002. (in Chinese)

[17] Zhang, P.L., Li, B., Wang, X.W., Liu, C.Y., Bi, W.J., Ma, H.Z. (2020). The loading characteristics of bulk coal in the middle trough and its influence on rigid body parts. Strojniški vestnik Journal of Mechanical Engineering, vol. 66, no. 2, p. 114-126, DOI:10.5545/sv-jme.2019.6267.

[18] Li, S., Zhu, Z.C., Lu, H., Shen, G. (2019). A system reliabilitybased design optimization for the scraper chain of scraper conveyors with dependent failure modes. Eksploatacja I 
Niezawodnosc-Maintenance and Reliability, vol. 21, no. 3, p. 392-402, DOI: 10.17531/ein.2019.3.5.

[19] Xue, H., Gu, S.P., Gong, X.Y., Ishihara, Z.Y., Tong, K.H. (2012). Analysis on structural integrity of defective mine round-link chains. Mining \& Processing Equipment, vol. 40, no. 12, p. 98101, D0I: 10.16816/j.cnki.ksjx.2012.12.028. (in Chinese)

[20] Zhao, L., Jin, X., Liu, X. (2020). Numerical research on wear characteristics of drum based on discrete element method (DEM). Engineering Failure Analysis, vol. 109, art. ID 104269, D0l:10.1016/j.engfailanal.2019.104269.

[21] Zhou, C., Yue, H., Li, Y., Zhang, M., Liu, J., Aijaz, S. (2019). A sphere filling algorithm for irregular aggregate particle generation based on non-linear optimization method. KSCE Journal of Civil Engineering, vol. 23, p. 120-129, D0l:10.1007/ s12205-018-0182-8.

[22] Richesson, S., Sahimi, M. (2019). Hertz-Mindlin theory of contacting grains and the effective-medium approximation for the permeability of deforming porous media. Geophysical
Research Letters, vol. 46, no. 14, p. 8039-8045, DOI:10.1029/2019g1083727.

[23] Hong, X.H. (2005). Mine Transportation and Lifting. China University of Mining and Technology Press, Xuzhou. (in Chinese)

[24] Liu, X., Wei, L. (2017). Vibration research on the polygonal effect of scraper conveyor. International Conference on Computer Technology, Electronics and Communication, p. 866-870, D0I:10.1109/ICCTEC.2017.00192.

[25] Bai, J., Xie, M.J. (2016). Scraper conveyor fault statistics and analysis of Shendong mining. Coal Mine Machinery, vol. 37, no. 11, p. 141-143, D0l:10.13436/J.mkjx.201611050. (in Chinese)

[26] Xia, R., Li, B., Wang, X.W., Yang, Z.J., Liu, L.P. (2019). Screening the main factors affecting the wear of the scraper conveyor chute using the Plackett-Burman method. Mathematical Problems in Engineering, vol. 2019, art. ID 1204091, DOI:10.1155/2019/1204091. 